\title{
A CONNECTION BETWEEN THE VOLUME FRACTIONS OF THE STIENEN MODEL AND THE DEAD LEAVES MODEL
}

\author{
MARIANNE MÅNSSON,* Chalmers University of Technology and Göteborg University
}

\begin{abstract}
The volume fraction of the intact grains of the dead leaves model with spherical grains of equal size is $2^{-d}$ in $d$ dimensions. This is the volume fraction of the original Stienen model. Here we consider some variants of these models: the dead leaves model with grains of a fixed convex shape and possibly random sizes and random orientations, and a generalisation of the Stienen model with convex grains growing at random speeds. The main result of this paper is that if the radius distribution in the dead leaves model equals the speed distribution in the Stienen model, then the volume fractions of the two models are the same in this case also. Furthermore, we show that for grains of a fixed shape and orientation, centrally symmetric sets give the highest volume fraction, while simplices give the lowest. If the grains are randomly rotated, then the volume fraction achieves its highest value only for spheres.
\end{abstract}

Keywords: Dead leaves model; Stienen model; Matérn hard core process; nonintersecting grains; volume fraction; convex grain

2000 Mathematics Subject Classification: Primary 60D05

Secondary 60G55; 52A22

\section{Introduction}

Two well-known random models for nonintersecting spheres are the intact grains of the dead leaves model and the Stienen model. The dead leaves model, which was introduced by Matheron (1968), is usually described in two dimensions; one can think of leaves falling randomly to the ground from time $-\infty$ to time 0 . The intact grains, or leaves, are then those which are intact at time 0 , when viewed from above. The leaves can be any compact objects, possibly of random shape, size, and orientation. A proper description of this model is given in Section 4.

The Stienen model was originally proposed in a materials science context by Stienen (1982). In his model, each point of a stationary Poisson process is the centre of a sphere with a diameter equal to the distance to its closest neighbour. Another way to determine the size of a particular sphere, say $A$, in the Stienen model, is to let all spheres grow at unit speed until the first collision with $A$, disregarding collisions between other spheres. The size of sphere $A$ at this collision is the size it is allocated in the Stienen model. The reason for giving this description is that it allows for natural generalisations of the model: one is to let the grains grow at random speeds, and another is to allow the grains to have shapes other than spherical.

The Stienen and dead leaves models are in many respects quite different. The germs of the Stienen model constitute a Poisson process, while this is not the case with the intact grains of the dead leaves model. In the patterns of the Stienen model, small spheres typically tend to

Received 24 February 2006; revision received 28 August 2006.

* Current address: Sagmastaregaten 1 i, SE 41680 Göteborg, Sweden. Email address: marianne@ math.chalmers.se 
appear in touching pairs and larger spheres tend to appear in isolation, while the probability of grains touching each other is 0 in the dead leaves model; if the falling leaves are of a fixed size, then this is clearly also the case for the intact leaves, while the sizes of the grains in the Stienen model are determined by the underlying Poisson process and by the growth speed distribution.

In the present paper, however, we focus on the similarity between the two models rather than on the differences. This similarity concerns the volume fraction, which, for stationary germ-grain models with nonintersecting grains, can be written as

$$
\rho=\lambda \bar{V}
$$

where $\lambda$ is the intensity of the germs and $\bar{V}$ denotes the mean volume of a typical grain. The main result of the paper is the following: if the radii of the falling leaves in the dead leaves model have the same distribution as the growth speeds of the grains in the Stienen model, then the two models have the same volume fraction. Furthermore, we present the radius distribution of the grains in both models and give upper and lower bounds on the volume fraction. The grains are assumed to have a given convex shape, but can have random sizes and orientations.

\section{Preliminaries}

The size of a sphere is often given in terms of its radius; in accordance with this we define the size of a convex grain $K \subset \mathbb{R}^{d}$ as being half its diameter, that is,

$$
\sup _{x, y \in K} \frac{|x-y|}{2},
$$

where $|\cdot|$ denotes the Euclidean distance. Let $\mathcal{C}^{d}$ denote the family of compact, convex sets $K$ that have interior points in $\mathbb{R}^{d}$, contain $o$ (the origin), and are of size 1 .

Let $l_{d}$ denote the $d$-dimensional Lebesgue measure. Furthermore, let $B_{d}(z, r)=\{x \in$ $\left.\mathbb{R}^{d}:|z-x| \leq r\right\}$ denote the $d$-dimensional ball of radius $r$ centred at $z$. The volume and the surface area of $B_{d}(o, 1)$ are denoted by $\kappa_{d}$ and $\omega_{d}$, respectively. Let $K(z, r)$ denote a set that has the same shape as $K$ but is translated by $z$ and has size $r>0$, that is, $K(z, r)=\{r y+z: y \in K\}$; then $l_{d}(K(z, r))=r^{d} l_{d}(K)$. Note that if $K \in \mathcal{C}^{d}$ then $K(o, 1)=K$.

For $A, B \subset \mathbb{R}^{d}$, Minkowski addition is defined by

$$
A \oplus B:=\{x+y: x \in A, y \in B\},
$$

which can also be written as

$$
A \oplus B=\{x: A \cap(\check{B}+x) \neq \varnothing\}
$$

where $\check{B}=-B$ denotes the reflection of $B$ at the origin. The volume of $x K \oplus y L$, where $x, y \in \mathbb{R}^{+}$and $K, L \subset \mathbb{R}^{d}$ are nonempty convex sets, can be written as

$$
l_{d}(x K \oplus y L)=\sum_{i=0}^{d}\left(\begin{array}{l}
d \\
i
\end{array}\right) x^{i} y^{d-i} V_{i, d-i}(K, L),
$$

where $V_{i, d-i}(K, L):=V(K, \ldots, K, L, \ldots, L)$ (with $K$ appearing $i$ times and $L$ appearing $d-i$ times) are the mixed volumes (mixed areas in $\mathbb{R}^{2}$ ) of $K$ and $L$ (a definition can be found in Schneider (1993)). Note the special cases $V_{d, 0}(K, L)=l_{d}(K)$ and $V_{0, d}(K, L)=l_{d}(L)$. 
The set

$$
K \oplus \check{K}=\left\{x \in \mathbb{R}^{d}: K \cap(K+\{x\}) \neq \varnothing\right\}
$$

is called the difference body of $K \subset \mathbb{R}^{d}$, and if $K$ is convex then

$$
2^{d} l_{d}(K) \leq l_{d}(K \oplus \check{K}) \leq\left(\begin{array}{c}
2 d \\
d
\end{array}\right) l_{d}(K)
$$

where the lower bound is attained if and only if $K$ is centrally symmetric and the upper bound is attained if and only if $K$ is a simplex. The left-hand inequality of (2.4) follows from the Brunn-Minkowski theorem, while the right-hand inequality is due to Rogers and Shephard (1957). Furthermore, if $K$ is a convex set then

$$
l_{d}(K) \leq V_{i, d-i}(K, \check{K}) \leq d^{\min \{i, d-i\}} l_{d}(K),
$$

with equality on the left-hand side if and only if $K$ is centrally symmetric or $i(d-i)=0$. On the right-hand side, there is equality in dimensions two and three if and only if $K$ is a triangle and a tetrahedron, respectively, or $i(d-i)=0$. For a proof of (2.5), see, e.g. Bonnesen and Fenchel (1948, p. 105). It was conjectured by Godbersen (1938) and Makai, Jr. (1974) that, furthermore,

$$
V_{i, d-i}(K, \check{K}) \leq\left(\begin{array}{l}
d \\
i
\end{array}\right) l_{d}(K),
$$

with equality if and only if $K$ is a simplex (see Schneider (1993, p. 412)).

Next we introduce the so-called intrinsic volumes, $V_{i}(K), i=1, \ldots, d$, for compact, convex $K \subset \mathbb{R}^{d}$, as follows:

$$
V_{i}(K):=\frac{1}{\kappa_{d-i}}\left(\begin{array}{l}
d \\
i
\end{array}\right) V_{i, d-i}\left(K, B_{d}(o, 1)\right) .
$$

Note that $V_{0}$ is identically equal to 1 and that $V_{d}$ is the volume. Furthermore, $2 V_{d-1}$ is the surface area and $2 \kappa_{d-1} V_{1} / \omega_{d}$ is the mean width.

\section{The Stienen model}

Two generalisations of the original Stienen model, in which spherical grains grow at the same speed, were proposed in Section 1:

- one with random growth speeds;

- one with nonspherical grains.

How the sizes of the spheres are determined in the original model can be described in two different ways: either the diameter of a sphere equals the distance from the centre of the sphere to the centre of its closest neighbour or, equivalently, a sphere is allocated the size it would attain if it grew from a germ until it met another sphere and all other spheres grew forever.

Using the latter description it is easy to generalise the model to have random growth speeds. Assume that all germs are assigned a random growth speed according to a distribution $F_{\mathrm{sp}}$, independently of each other and of the Poisson process. The sizes of the grains are determined as in the original model, the only change being that they now grow with random speeds. The second of the proposed generalisations is also easily explained using the second description of how the sizes are determined: here it is nonspherical grains of possibly different orientations that grow until the first collision with another grain. 
If all grains have the same shape as $K \in \mathcal{C}^{d}$, then the volume fraction of the Stienen model is

$$
\rho=\lambda \mathrm{E}\left[l_{d}(K(o, R))\right]=\lambda l_{d}(K) \mathrm{E}\left[R^{d}\right],
$$

where $R$ denotes the random size (half the diameter) of a typical grain and $\lambda$ is the intensity of the underlying Poisson process. Thus, we need to find the distribution of $R$ in order to determine the volume fraction. This is what will concern us in the remainder of this section, and will be considered in two different cases: in the first subsection we consider grains of fixed orientation, while in the second we consider randomly oriented grains.

\subsection{Fixed orientation}

In the original $d$-dimensional Stienen model a sphere grows halfway to its nearest neighbour, and since we start with a Poisson point process it is clear that the radius of a sphere at the origin is larger than $r$ if there are no points in $B_{d}(o, 2 r)$. Hence,

$$
\mathrm{P}(R>r)=\exp \left\{-\lambda \kappa_{d}(2 r)^{d}\right\},
$$

which yields

$$
\mathrm{E}\left[R^{d}\right]=\left(2^{d} \lambda \kappa_{d}\right)^{-1} .
$$

Inserting (3.2) into (3.1) gives the volume fraction $\rho=2^{-d}$.

This result is easily generalised to grains of the same shape and orientation as $K \in \mathcal{C}^{d}$. For spherical sets, the radius of a sphere is larger than $r$ if there are no points in the set

$$
B_{d}(o, 2 r)=\left\{x \in \mathbb{R}^{d}: B_{d}(o, r) \cap B_{d}(x, r) \neq \varnothing\right\} .
$$

For nonspherical grains, we need to replace this set by

$$
\left\{x \in \mathbb{R}^{d}: K(o, r) \cap K(x, r) \neq \varnothing\right\}=K(o, r) \oplus \check{K}(o, r)
$$

(which exactly follows from (2.1)). The distribution function and volume fraction then follow as above.

Now we let the grains grow with random speeds which are independent of each other and assume the grains to have the same shape and orientation as $K \in \mathcal{C}^{d}$. For a distribution function $F$, consider

$$
\begin{aligned}
\Lambda_{\mathrm{fix}}(K, r, F) & :=\int_{0}^{\infty} l_{d}(K(o, r) \oplus \check{K}(o, s)) \mathrm{d} F(s) \\
& =\sum_{i=0}^{d}\left(\begin{array}{l}
d \\
i
\end{array}\right) r^{i} V_{i, d-i}(K, \check{K}) \int_{0}^{\infty} s^{d-i} \mathrm{~d} F(s),
\end{aligned}
$$

where the equality follows from (2.2), and assume that the $d$ th moment of $F$ exists. First we determine the distribution function of the final size of a typical grain, letting $F_{\text {sp }}$ denote the distribution of the growth speed.

Theorem 3.1. Assume that the grains have the same shape and orientation as $K \in \mathcal{C}^{d}$ and that the dth moment of $F_{\mathrm{sp}}$ exists. Then the following results hold for the Stienen model with grains growing with random speeds.

(i) The distribution function of the final size of a typical grain is

$$
F(r)=1-\int_{0}^{\infty} \exp \left\{-\lambda(r / s)^{d} \Lambda_{\mathrm{fix}}\left(K, s, F_{\mathrm{sp}}\right)\right\} \mathrm{d} F_{\mathrm{sp}}(s) .
$$


(ii) The volume fraction is

$$
\rho=l_{d}(K) \int_{0}^{\infty} s^{d} \Lambda_{\mathrm{fix}}\left(K, s, F_{\mathrm{sp}}\right)^{-1} \mathrm{~d} F_{\mathrm{sp}}(s) .
$$

Proof. (i) Assume that there is a grain at the origin which grows at speed $s$. The size of this grain is smaller than $r$ if it stops growing before the time $r / s$. For this to happen there must be at least one point of the original Poisson process with some speed $h$ and some position $z$ which collides with the grain at the origin before time $r / s$, that is, such that

$$
K(o, r) \cap K(z, h r / s) \neq \varnothing .
$$

Since the speeds are independent of each other, the positions of the grains which can prevent the size of the grain at the origin exceeding $r$ can be seen as an independent thinning of the original Poisson process, which results in an inhomogeneous Poisson process on $\mathbb{R}^{d} \times \mathbb{R}^{+}$with intensity

$$
\lambda \mathbf{1}_{\{x \in\{z: K(o, r) \cap K(z, h r / s) \neq \varnothing\}\}} \mathrm{d} x \mathrm{~d} F_{\mathrm{sp}}(h)=\lambda \mathbf{1}_{\{x \in K(o, r) \oplus \check{K}(o, h r / s)\}} \mathrm{d} x \mathrm{~d} F_{\mathrm{sp}}(h),
$$

where the equality follows from (2.3). The expected total number of points of this process is

$$
\begin{aligned}
\lambda \int_{0}^{\infty} \int_{K(o, r) \oplus \check{K}(o, h r / s)} \mathrm{d} x \mathrm{~d} F_{\mathrm{sp}}(h) & =\lambda \sum_{i=0}^{d}\left(\begin{array}{c}
d \\
i
\end{array}\right) r^{i} V_{i, d-i}(K, \check{K}) \int_{0}^{\infty}\left(\frac{h r}{s}\right)^{d-i} \mathrm{~d} F_{\mathrm{sp}}(h) \\
& =\lambda\left(\begin{array}{c}
r \\
s
\end{array}\right)^{d} \sum_{i=0}^{d}\left(\begin{array}{c}
d \\
i
\end{array}\right) s^{i} V_{i, d-i}(K, \check{K}) \int_{0}^{\infty} h^{d-i} \mathrm{~d} F_{\mathrm{sp}}(h),
\end{aligned}
$$

using (2.2) in the first equality. The radius of a grain at the origin growing at speed $s$ exceeds $r$ if there are no points at all in this Poisson process. The proof is concluded by integrating over all possible growth speeds.

(ii) From (i) it follows that

$$
\mathrm{E}\left[R^{d}\right]=\frac{1}{\lambda} \int_{0}^{\infty} s^{d} \Lambda_{\mathrm{fix}}\left(K, s, F_{\mathrm{sp}}\right)^{-1} \mathrm{~d} F_{\mathrm{sp}}(s),
$$

and we obtain the volume fraction by inserting this expectation into (3.1).

Note that if all grains grow at the same speed, say $s_{0}$, then

$$
\Lambda_{\text {fix }}\left(K, s_{0}, F_{\text {sp }}\right)=s_{0}^{d} l_{d}(K \oplus \check{K})
$$

and we obtain the following result.

Corollary 3.1. In the Stienen model with convex grains that have equal shape and orientation and grow at the same speed, the distribution function of the size of a typical grain is given by

$$
F(r)=1-\exp \left\{-\lambda r^{d} \Lambda_{\text {fix }}(K)\right\},
$$

where

$$
\Lambda_{\mathrm{fix}}(K):=l_{d}(K \oplus \check{K})=\sum_{i=0}^{d}\left(\begin{array}{l}
d \\
i
\end{array}\right) V_{i, d-i}(K, \check{K}) .
$$

The volume fraction is

$$
\rho=\frac{l_{d}(K)}{\Lambda_{\text {fix }}(K)}
$$




\subsection{Random orientations}

In addition to letting the grains grow at different speeds, we can also let them have different orientations. Let $S O(d)$ denote the group of rotations about the origin. By a uniformly distributed rotation we mean an element from $S O(d)$, chosen according to the Haar measure $v$, with $v(S O(d))=1$ (see, e.g. Schneider and Wieacker (1993) for details). Here we let the grains be rotated according to this uniform distribution, and we let the rotations be independent of each other and of the positions of the grains. Let the sizes of the grains be determined as previously.

This yields another inhomogeneous Poisson process consisting of germs which can prevent the size of the grain at the origin exceeding $r$. This process is defined on $\mathbb{R}^{d} \times \mathbb{R}^{+} \times S O(d)$ and has the intensity measure

$$
\lambda \mathbf{1}_{\{x \in K(o, r) \oplus \vartheta K(o, h r / s)\}} \mathrm{d} x \mathrm{~d} F_{\mathrm{sp}}(h) v(\mathrm{~d} \vartheta) .
$$

If we let

$$
\begin{aligned}
\Lambda_{\mathrm{rot}}(K, r, F) & :=\int_{0}^{\infty} \int_{S O(d)} l_{d}(K(o, r) \oplus \vartheta K(o, y)) v(\mathrm{~d} \vartheta) \mathrm{d} F(y) \\
& =\frac{1}{\kappa_{d}} \sum_{k=0}^{d} \kappa_{k} \kappa_{d-k}\left(\begin{array}{l}
d \\
k
\end{array}\right)^{-1} r^{k} V_{k}(K) V_{d-k}(K) \int_{0}^{\infty} y^{d-k} \mathrm{~d} F(y),
\end{aligned}
$$

where we have used the generalised Steiner formula (see, e.g. Weil and Wieacker (1993, p. 1407)) in the second equality, the expected total number of points of this Poisson process is

$$
\lambda \int_{0}^{\infty} \int_{S O(d)} \int_{K(o, r) \oplus \vartheta K(o, h r / s)} \mathrm{d} x v(\mathrm{~d} \vartheta) \mathrm{d} F_{\mathrm{sp}}(h)=\lambda\left(\frac{r}{s}\right)^{d} \Lambda_{\mathrm{rot}}\left(K, s, F_{\mathrm{sp}}\right) .
$$

The proof is concluded by integrating over all possible growth speeds.

Theorem 3.2. Theorem 3.1 holds in the case of random orientations if $\Lambda_{\text {fix }}$ is replaced by $\Lambda_{\text {rot }}$, defined in (3.5).

Corollary 3.2. If all grains grow at the same speed, then

$$
\rho=\frac{l_{d}(K)}{\Lambda_{\text {rot }}(K)}
$$

where

$$
\Lambda_{\mathrm{rot}}(K):=\int_{S O(d)} l_{d}(K \oplus \vartheta K) v(\mathrm{~d} \vartheta)=\frac{1}{\kappa_{d}} \sum_{k=0}^{d} \kappa_{k} \kappa_{d-k}\left(\begin{array}{l}
d \\
k
\end{array}\right)^{-1} V_{k}(K) V_{d-k}(K)
$$

\section{The dead leaves model}

The original dead leaves model was introduced by Matheron (1968) and is a random tessellation of space as well as being a model for nonintersecting sets. It can be defined as follows. Consider a stationary Poisson process $\left\{\left(x_{i}, t_{i}\right)\right\}$ with unit intensity in $\mathbb{R}^{d} \times(-\infty, 0]$. Interpret $t_{i}$ as the arrival time of the point $x_{i} \in \mathbb{R}^{d}$. Let $d$-dimensional, possibly random, compact grains be placed at the points $x_{i}$ sequentially in time, in such a way that a new grain 
might delete portions of 'older' ones. At time $t=0$ the space $\mathbb{R}^{d}$ is completely covered, and the grains which are not completely deleted constitute a tessellation of $\mathbb{R}^{d}$ which is called the dead leaves model.

The grains which are intact, that is, not intersected by any later grains, constitute a model of nonintersecting grains. Note that the union of the intact grains constitute a random closed set in $\mathbb{R}^{d}$. The intact grains of the dead leaves model can also be considered the limit of a generalisation of one of Matérn's hard core models introduced in Månsson and Rudemo (2002). This generalisation is constructed as follows.

1. First generate a Poisson process with constant intensity in $\mathbb{R}^{d}$. At the points of this process place independent grains of a given shape, but of possibly random sizes and orientations. Furthermore, to each grain give a weight, according to a continuous distribution. The weights are independent of each other and of everything else.

2. Then thin the process by letting all pairs of grains which intersect 'compete': a grain is kept if it has the higher weight in all pairwise comparisons with the grains it intersects.

The original Poisson process together with the grains can be seen as a proposal model. In accordance with this, we let $\lambda_{\mathrm{pr}}$ and $F_{\mathrm{pr}}$ denote the proposal intensity and proposal distribution function of the sizes of the grains, respectively, before thinning. The intensity measure and distribution function of the sizes in the thinned process will be denoted by $\lambda$ and $F$, respectively.

Let $\Psi_{T}$ denote this model with intensity $\lambda_{\mathrm{pr}}=T$ and weights uniformly distributed on $[-T, 0]$. Then the points of the original Poisson process together with the weights can be regarded as a Poisson process with unit intensity in $\mathbb{R}^{d} \times[-T, 0]$. If we let $\Psi_{i}, i=1,2, \ldots$, be based on the same Poisson process in $\mathbb{R}^{d} \times(-\infty, 0]$ and think of the weights as arrival times, it follows that $\Psi_{i} \subset \Psi_{i+1}$, and the limiting random set $\bigcup_{i=1}^{\infty} \Psi_{i}$ equals the intact grains of the dead leaves model. Results for $\Psi_{T}$ can now be used to obtain results for the intact grains of the dead leaves model.

The dead leaves model and generalisations of it, for instance the colour dead leaves, have been studied in a number of papers by Jeulin; see, e.g. Jeulin (1997). Results on the intensity and size distributions of the intact grains can be found in Jeulin (1989). The connection between Matérn's second hard core model and the dead leaves model in the case of fixed-sized spheres was noted by Stoyan and Schlater (2000). In Andersson et al. (2006) some aspects of the volume fraction of the dead leaves model were considered.

The following results for the generalisation of Matérn's model can be found in, or follow directly from, Månsson and Rudemo (2002).

Lemma 4.1. Assume that the grains have the same shape as $K \in \mathcal{C}^{d}$ and that the dth moment of $F_{\mathrm{pr}}$ exists. Let $\Lambda(K, r)=\Lambda_{\mathrm{fix}}(K, r)$ if the orientation is fixed and let $\Lambda(K, r, F)=$ $\Lambda_{\text {rot }}(K, r, F)$ otherwise, where $\Lambda_{\mathrm{fix}}(K, r, F)$ and $\Lambda_{\mathrm{rot}}(K, r, F)$ are as given in (3.3) and (3.5), respectively.

(i) The intensity is

$$
\lambda=\int_{0}^{\infty} \frac{1-\exp \left\{-\lambda{ }_{\mathrm{pr}} \Lambda\left(K, r, F_{\mathrm{pr}}\right)\right\}}{\Lambda\left(K, r, F_{\mathrm{pr}}\right)} \mathrm{d} F_{\mathrm{pr}}(r) .
$$

(ii) The size distribution of the grains is

$$
F(r)=1-\lambda^{-1} \int_{r}^{\infty} \frac{1-\exp \left\{-\lambda \mathrm{pr} \Lambda\left(K, s, F_{\mathrm{pr}}\right)\right\}}{\Lambda\left(K, s, F_{\mathrm{pr}}\right)} \mathrm{d} F_{\mathrm{pr}}(s) .
$$


(iii) The volume fraction is

$$
\rho=\int_{0}^{\infty} r^{d} \frac{1-\exp \left\{-\lambda{ }_{\mathrm{pr}} \Lambda\left(K, r, F_{\mathrm{pr}}\right)\right\}}{\Lambda\left(K, r, F_{\mathrm{pr}}\right)} \mathrm{d} F_{\mathrm{pr}}(r) .
$$

Letting $\lambda_{\text {pr }}$ tend to $\infty$ in the above lemma immediately gives the following theorem.

Theorem 4.1. Assume that the grains have the same shape as $K \in \mathrm{C}^{d}$ and that the dth moment of $F_{\mathrm{pr}}$ exists. Let $\Lambda(K, r, F)=\Lambda_{\mathrm{fix}}(K, r, F)$ if the orientation is fixed and let $\Lambda(K, r, F)=$ $\Lambda_{\text {rot }}(K, r, F)$ otherwise, where $\Lambda_{\text {fix }}(K, r, F)$ and $\Lambda_{\text {rot }}(K, r, F)$ are as given in (3.3) and (3.5), respectively. The following results hold for the intact grains of the dead leaves model.

(i) The intensity is

$$
\lambda=\int_{0}^{\infty}\left(\Lambda\left(K, r, F_{\mathrm{pr}}\right)\right)^{-1} \mathrm{~d} F_{\mathrm{pr}}(r) .
$$

(ii) The size distribution of the grains is

$$
F(r)=1-\lambda^{-1} \int_{r}^{\infty} \Lambda\left(K, s, F_{\mathrm{pr}}\right)^{-1} \mathrm{~d} F_{\mathrm{pr}}(s) .
$$

If the original size distribution is continuous with density $f_{\mathrm{pr}}$, then the distribution of the sizes is also continuous, with density

$$
f(r)=\frac{f_{\mathrm{pr}}(r)}{\Lambda\left(K, r, F_{\mathrm{pr}}\right) \lambda} .
$$

(iii) The volume fraction is

$$
\rho=l_{d}(K) \int_{0}^{\infty} r^{d} \Lambda\left(K, r, F_{\mathrm{pr}}\right)^{-1} \mathrm{~d} F_{\mathrm{pr}}(r) .
$$

In particular, if the grain size is fixed then

$$
\rho=\frac{l_{d}(K)}{\Lambda(K)},
$$

where $\Lambda(K)$ equals $\Lambda_{\mathrm{fix}}(K)$ if the orientation is fixed and equals $\Lambda_{\mathrm{rot}}(K)$ if the orientations are random. The functions $\Lambda_{\mathrm{fix}}(K)$ and $\Lambda_{\mathrm{rot}}(K)$ are as defined in (3.4) and (3.6), respectively.

Comparing Theorems 3.1 and 4.1, we have the following result.

Theorem 4.2. If the growth speed distribution in the Stienen model, $F_{\mathrm{sp}}$, and the grain size distribution before thinning in the dead leaves model, $F_{\mathrm{pr}}$, are equal, then the volume fractions are equal in the two models.

\section{Bounds on the volume fraction}

The volume fraction depends on the shape of the grains. For instance, in two dimensions the area fraction is $\frac{1}{4}$ for discs and $\frac{1}{6}$ for triangles of a fixed size and orientation. In the formulae in Theorems 3.1 and 4.1 it can be seen that the volume fraction depends on the shape through the mixed volumes if the orientation is fixed. If the orientations are random then the volume fraction depends on the shape through intrinsic volumes rather than through mixed volumes. 


\subsection{Fixed orientation}

We will now use the inequalities concerning mixed volumes in Section 2 to give upper and lower bounds on the volume fraction.

Theorem 5.1. (i) Let the grains have the same shape and orientation as $K \in \mathcal{C}^{d}$. Let $F_{\mathrm{pr}}$ denote the proposal distribution of the grain sizes in the dead leaves model and the growth speed distribution in the Stienen model, and assume that the dth moment of $F_{\mathrm{pr}}$ exists. Then the volume fraction of these models has the following bounds:

$$
\begin{aligned}
& \rho \geq \int_{0}^{\infty}\left(\sum_{i=0}^{d}\left(\begin{array}{l}
d \\
i
\end{array}\right) r^{i-d} d^{\min \{i, d-i\}} \int_{0}^{\infty} s^{d-i} \mathrm{~d} F_{\mathrm{pr}}(s)\right)^{-1} \mathrm{~d} F_{\mathrm{pr}}(r), \\
& \rho \leq \int_{0}^{\infty}\left(\sum_{i=0}^{d}\left(\begin{array}{l}
d \\
i
\end{array}\right) r^{i-d} \int_{0}^{\infty} s^{d-i} \mathrm{~d} F_{\mathrm{pr}}(s)\right)^{-1} \mathrm{~d} F_{\mathrm{pr}}(r) .
\end{aligned}
$$

The upper bound is attained if and only if $K$ is centrally symmetric. For $d=2$ and $d=3$, the lower bound is attained if and only if $K$ is a triangle and a tetrahedron, respectively. Furthermore, if the conjectured inequality (2.6) is true, then

$$
\rho \geq \int_{0}^{\infty}\left(\sum_{i=0}^{d}\left(\begin{array}{l}
d \\
i
\end{array}\right)^{2} r^{i-d} \int_{0}^{\infty} s^{d-i} \mathrm{~d} F_{\mathrm{pr}}(s)\right)^{-1} \mathrm{~d} F_{\mathrm{pr}}(r),
$$

with equality if and only if $K$ is a simplex.

(ii) If the grain sizes in the dead leaves model and the growth speed in the Stienen model are fixed, then the volume fraction has the following bounds:

$$
\left(\begin{array}{c}
2 d \\
d
\end{array}\right)^{-1} \leq \rho \leq 2^{-d}
$$

The upper bound is attained if and only if $K$ is centrally symmetric, and the lower bound is attained if and only if $K$ is a simplex.

Proof. (i) This result follows from Theorem 3.1, (2.5), and (2.6).

(ii) This result follows from Corollary 3.1 and (2.4).

\subsection{Random orientations}

We now present upper bounds on the volume fraction when the orientations of the grains are random. The lower bound is 0 , since for any fixed volume there exist sets with this volume and arbitrarily large intrinsic volumes of lower dimensions, meaning that $\Lambda_{\text {rot }}$ can be arbitrarily large. For instance, in two dimensions there are rectangles of area 1 and arbitrarily large perimeter.

Theorem 5.2. (i) Assume that the grains are independently and uniformly rotated and have the same shape as $K \in \mathrm{C}^{d}$. Let $F_{\mathrm{pr}}$ denote the proposal distribution of the grain sizes in the dead leaves model and the growth speed distribution in the Stienen model, and assume that the $d$ th moment of $F_{\mathrm{pr}}$ exists. Then the volume fractions in these models have the following bound, with equality if and only if $K$ is a sphere:

$$
\rho \leq \int_{0}^{\infty}\left(\sum_{i=0}^{d}\left(\begin{array}{l}
d \\
i
\end{array}\right) r^{i-d} \int_{0}^{\infty} s^{d-i} \mathrm{~d} F_{\mathrm{pr}}(s)\right)^{-1} \mathrm{~d} F_{\mathrm{pr}}(r) .
$$


(ii) If the grain sizes in the dead leaves model and the growth speed in the Stienen model are fixed, then

$$
\rho \leq 2^{-d}
$$

with equality if and only if $K$ is a sphere.

Proof. (i) From the Brunn-Minkowski theorem it follows that

$$
l_{d}(\{x: K(o, r) \cap \vartheta K(x, y) \neq \varnothing\}) \geq(r+y)^{d} l_{d}(K),
$$

with equality if and only if $K$ and $\vartheta(\check{K})$ are translates of one another. Hence,

$$
\begin{aligned}
\int_{0}^{\infty} & \int_{S O(d)} l_{d}(\{x: K(o, r) \cap \vartheta K(x, y) \neq \varnothing\}) v(\mathrm{~d} \vartheta) \mathrm{d} F_{\mathrm{pr}}(y) \\
& \geq \int_{0}^{\infty}(r+y)^{d} l_{d}(K) \mathrm{d} F_{\mathrm{pr}}(y) \\
& =l_{d}(K) \sum_{i=0}^{d}\left(\begin{array}{l}
d \\
i
\end{array}\right) r^{i} \int_{0}^{\infty} y^{d-i} \mathrm{~d} F_{\mathrm{pr}}(y),
\end{aligned}
$$

with equality if and only if $K$ is a sphere, since $K$ and $\vartheta(\check{K})$ are translates of one another for all $\vartheta \in S O(d)$ if and only if $K$ is a sphere. The result of (i) then follows from Theorem 4.1 and (3.5).

(ii) This result follows immediately from that of (i).

Unlike in the case of fixed orientations, the centrally symmetric sets do not all behave in the same way now: here spheres are the only sets for which the upper bound of the volume fraction is attained. Furthermore, all triangles and tetrahedra do not give the same volume fraction when the orientations are random.

\subsection{Discs, triangles, and other extremal sets}

Finally, in this subsection we calculate the volume fraction of the Stienen model with grains growing at equal speeds and that of the dead leaves model with fixed-sized grains of the following shapes: discs, squares, and equilateral triangles in two dimensions, and spheres, cubes, and regular tetrahedra in three dimensions. For fixed orientations we have, according to (5.1),

$$
\frac{1}{6} \leq \rho \leq \frac{1}{4} \quad \text { if } d=2 \quad \text { and } \quad \frac{1}{20} \leq \rho \leq \frac{1}{8} \quad \text { if } d=3,
$$

with equality on the right-hand side of each inequality if and only if $K$ is centrally symmetric (for instance for discs, spheres, squares, and cubes) and equality on the left-hand side of each if and only if $K$ is a triangle and a tetrahedron, respectively.

In the case of random orientations it follows from Theorem 4.1 that

$$
\rho= \begin{cases}\frac{l_{2}(K)}{l_{2}(K) 2+S_{1}(K)^{2} /(2 \pi)} & \text { if } d=2, \\ \frac{l_{3}(K)}{l_{3}(K) 2+S_{2}(K) \bar{b}(K)} & \text { if } d=3,\end{cases}
$$

where $S_{1}$ is the perimeter, $S_{2}$ is the surface area, and $\bar{b}$ is the mean width. All quantities are straightforward to calculate, except perhaps those for the tetrahedron, which were 
TABLE 1: The volume fraction in some special cases.

\begin{tabular}{llcc}
\hline$d$ & Shape & Fixed orientation & Random orientation \\
\hline 2 & Disc & $\frac{1}{4}$ & $\frac{1}{4}$ \\
2 & Square & $\frac{1}{4}$ & $(2+8 / \pi)^{-1} \approx 0.22$ \\
2 & Equilateral triangle & $\frac{1}{6}$ & $\sqrt{3}(2 \sqrt{3}+18 / \pi)^{-1} \approx 0.19$ \\
3 & Sphere & $\frac{1}{8}$ & $\frac{1}{8}$ \\
3 & Cube & $\frac{1}{8}$ & $\frac{1}{11}$ \\
3 & Regular tetrahedron & $\frac{1}{20}$ & $\left(2+18 \sqrt{\frac{3}{2}}\left(1-\arccos \frac{1}{3} / \pi\right)\right)^{-1} \approx 0.068$ \\
\hline
\end{tabular}

given in Månsson and Rudemo (2002) as follows: $l_{3}(K)=2 \sqrt{2} / 3, S_{2}(K)=4 \sqrt{3}$, and $\bar{b}(K)=(3 / \pi)\left(\pi-\arccos \frac{1}{3}\right)$. The volume fractions are summarised in Table 1 .

Note that for squares and cubes the volume fraction is lower if the grains are randomly rotated than if they have a fixed orientation, and vice versa for triangles and tetrahedra.

\section{Ideas for future work}

In this paper the orientation of the grains in the original process is either fixed or uniformly distributed. A natural generalisation would be to let the grains have some anisotropic orientation distribution. As pointed out by a referee, Theorem 3.2 should still hold in this case with $\Lambda_{\text {rot }}$ defined as in (3.5), if the integration is performed with respect to the orientations. However, the generalised Steiner formula cannot be used. With this generalisation, there are many questions to consider, for example what distribution gives the highest volume fraction for a given shape. For triangles a guess is that a distribution assigning one of two opposite directions with equal probabilities would do so. Another issue is the connection between the orientation distributions before and after thinning.

\section{Acknowledgements}

I would like to thank the referees for valuable comments on, and corrections to, the first version of this paper. I am also indebted to one of the referees for the suggestion concerning anisotropic orientation distributions which is discussed in Section 6.

\section{References}

Andersson, J., Häggström, O. And MÅnsson, M. (2006). The volume fraction of a non-overlapping germ-grain model. Electron. Commun. Prob. 11, 78-88.

Bonnesen, T. And Fenchel, W. (1948). Theorie der Konvexen Körper. Springer, Berlin.

Godbersen, C. (1938). Der Satz vom Vektorbereich in Räumen beliebiger Dimensionen. Doctoral Thesis, University of Göttingen.

JEulin, D. (1989). Morphological modeling of images by sequential random functions. Signal Process. 16, $403-431$. Jeulin, D. (1997). Dead leaves models: from space tessellation to random functions. In Proc. Internat. Symp. Adv. Theory Appl. Random Sets (Fontainebleu, 1996), ed. D. Jeulin, World Scientific, River Edge, NJ, pp. 137-156.

MAKAI, E., JR (1974). Research problem. Periodica Math. Hung. 5, 353-354.

Månsson, M. and Rudemo, M. (2002). Random patterns of nonoverlapping convex grains. Adv. Appl. Prob. 34, 718-738.

Matheron, G. (1968). Schéma booléen séquentiel de partition aléatoire. Res. Rep. N83, Centre de Morphologie Mathématique, École des Mines de Paris.

Rogers, C. A. And Shephard, G. C. (1957). The difference body of a convex body. Arch. Math. 8, 220-233. 
SchneIder, R. (1993). Convex Bodies: the Brunn-Minkowski Theory. Cambridge University Press.

Schneider, R. AND Wieacker, J. A. (1993). Integral geometry. In Handbook of Convex Geometry, Vol. B, eds P. M. Gruber and J. M. Wills, North-Holland, Amsterdam, pp. 1349-1390.

Stienen, H. (1982). Die Vergroeberung von Karbiden in reinen Eisen-Kohlenstoff Staehlen. Doctoral Thesis, RWTH Aachen.

Stoyan, D. And Schlater, M. (2000). Random sequential adsorption: relationship to dead leaves and characterization of variability. J. Statist. Phys. 100, 969-979.

Weil, W. AND Wieacker, J. A. (1993). Stochastic geometry. In Handbook of Convex Geometry, Vol. B, eds P. M. Gruber and J. M. Wills, North-Holland, Amsterdam, pp. 1391-1438. 